\title{
Prevention of venous thromboembolism in hospitalized acutely ill medical patients: focus on the clinical utility of (low-dose) fondaparinux
}

This article was published in the following Dove Press journal:

Drug Design, Development and Therapy

12 September 2013

Number of times this article has been viewed

\author{
Marcello Di Nisio ${ }^{1,2}$ \\ Ettore Porreca ${ }^{3}$ \\ 'Department of Medical, Oral and \\ Biotechnological Sciences, University \\ G D'Annunzio of Chieti-Pescara, \\ Chieti, Italy; ${ }^{2}$ Department of Vascular \\ Medicine, Academic Medical Center, \\ Amsterdam, The Netherlands; \\ ${ }^{3}$ Department of Medicine and \\ Aging, Centre for Aging Sciences, \\ Internal Medicine Unit, University G \\ D'Annunzio Foundation, Chieti, Italy
}

Correspondence: Marcello Di Nisio Department of Medical, Oral and Biotechnological Sciences, University G D'Annunzio of Chieti-Pescara, via dei Vestini 3I, 660I3 Chieti, Italy

Tel +393283290020

Fax +39087135 7361

Email mdinisio@unich.it
Abstract: Venous thromboembolism (VTE) is a frequent complication among acutely ill medical patients hospitalized for congestive heart failure, acute respiratory insufficiency, rheumatologic disorders, and acute infectious and/or inflammatory diseases. Based on robust data from randomized controlled studies and meta-analyses showing a reduced incidence of VTE by $40 \%$ to about $60 \%$ with pharmacologic thromboprophylaxis, prevention of VTE with low molecular weight heparin (LMWH), unfractionated heparin (UFH), or fondaparinux is currently recommended in all at-risk hospitalized acutely ill medical patients. In patients who are bleeding or are at high risk for major bleeding, mechanical prophylaxis with graduated compression stockings or intermittent pneumatic compression may be suggested. Thromboprophylaxis is generally continued for 6 to 14 days or for the duration of hospitalization. Selected cases could benefit from extended thromboprophylaxis beyond this period, although the risk of major bleeding remains a concern, and additional studies are needed to identify patients who may benefit from prolonged prophylaxis. For hospitalized acutely ill medical patients with renal insufficiency, a low dose (1.5 mg once daily) of fondaparinux or prophylactic LMWH subcutaneously appears to have a safe profile, although proper evaluation in randomized studies is lacking. The evidence on the use of prophylaxis for VTE in this latter group of patients, as well as in those at higher risk of bleeding complications, such as patients with thrombocytopenia, remains scarce. For critically ill patients hospitalized in intensive care units with no contraindications, LMWH or UFH are recommended, with frequent and careful assessment of the risk of bleeding. In this review, we discuss the evidence for use of thromboprophylaxis for VTE in acutely ill hospitalized medical patients, with a focus on (low-dose) fondaparinux.

Keywords: venous thromboembolism, medical patient, hospitalization, fondaparinux, heparin

\section{Introduction}

Venous thromboembolism (VTE), defined as deep venous thrombosis (DVT) or pulmonary embolism (PE), complicates the course of acute medical diseases in as many as $10 \%-20 \%$ of hospitalized patients. ${ }^{1}$ Groups of medical patients at increased risk for VTE include those with congestive heart failure (New York Heart Association Class III or IV), acute respiratory insufficiency, rheumatologic disorders, acute infectious diseases, inflammatory bowel disease, and arterial thrombotic disease, namely, acute myocardial infarction or ischemic stroke (Table 1). ${ }^{2-10}$ Additional risk factors predisposing to VTE in medical patients are a positive history of VTE, cancer, advanced age, and prolonged immobility. 2,6,11-13 The risk of VTE differs across the spectrum of hospitalized medical patients, and quantitative risk prediction scores were developed 
Table I Acute medical illnesses and predisposing risk factors for venous thromboembolism in hospitalized medical patients

\begin{tabular}{l} 
Acute medical illnesses \\
Acute congestive heart failure (NYHA Class III or IV) \\
Acute respiratory disease \\
Acute infectious disease \\
Acute rheumatic disease \\
Inflammatory bowel disease \\
Ischemic stroke \\
Acute myocardial infarction \\
Predisposing factors \\
Previous venous thromboembolism \\
Older age (especially $>70$ years) \\
Marked reduction of mobility \\
Malignancy \\
Recent ( $\leq$ I month) trauma and/or surgery \\
Obesity (BMI > 30) \\
Inherited or acquired thrombophilic states (eg, antiphospholipid \\
syndrome) \\
Varicose veins \\
Estrogen therapy or pregnancy \\
Chronic kidney disease \\
Invasive procedures (eg, central venous catheterization) \\
\hline Abreviations: BMI body mass index; NYHA New York Heart Association
\end{tabular}

Abbreviations: BMI, body mass index; NYHA, New York Heart Association.

to appreciate the cumulative effect of VTE risk factors, and to help discriminate between patients at high versus low risk of VTE. ${ }^{14}$ The latest American College of Chest Physicians guidelines adopted the Padua Prediction Score developed by Barbar et al, and recommended VTE prophylaxis in patients classified at high risk according to the score. ${ }^{2,15}$ The incidence of VTE was $2.2 \%$ in patients with and $11.0 \%$ in those without thromboprophylaxis, while VTE occurred in only $0.3 \%$ in patients classified as being at low risk. ${ }^{15}$ Although none of the prediction scores proposed thus far appear to have any clear advantage, the key feature of all is that risk increases rapidly with summation of risk factors. None of the predictive scores gained widespread acceptance and none is routinely implemented in clinical practice, which may depend on the failure of the validation studies to replicate the ability of such models to stratify the risk of VTE. ${ }^{14}$ For this reason, and for ease of use, experts in the field and some clinical guidelines simply divide medical patients into at-risk, for whom VTE prophylaxis is recommended, and not-at-risk categories based on the presence of at least one or two risk factors. ${ }^{16,17}$

In this review, we discuss the evidence on the use of thromboprophylaxis for VTE in acutely ill hospitalized medical patients with a focus on (low-dose) fondaparinux.

\section{VTE prophylaxis}

A number of randomized controlled trials (RCTs) and subsequent meta-analyses demonstrated that pharmacologic thromboprophylaxis in medical patients significantly reduces the incidence of VTE by $40 \%$ to about $60 \% .^{2-4,18-21}$ These findings prompted panels of experts and international guidelines to recommend thromboprophylaxis with either low molecular weight heparin (LMWH), unfractionated heparin (UFH), or fondaparinux in medical patients at increased risk of VTE (Table 2). ${ }^{1-4}$ Direct comparisons with UFH suggested that LMWH is associated with a $32 \%$ lower risk of DVT and of major bleeding. ${ }^{2,4}$ Given the superior adverse effect profile and the ease of use, LMWH or fondaparinux may be preferred over UFH, which could be considered for patients in whom LMWH is contraindicated.

Two recent studies evaluated the new oral anticoagulants, apixaban and rivaroxaban, against a standard course of LMWH (enoxaparin) as thromboprophylaxis in hospitalized medical patients. ${ }^{22,23}$ In the ADOPT (Apixaban Dosing to Optimize Protection from Thrombosis) trial, an extended course of apixaban was associated with a nonsignificant decrease in VTE and VTE-related mortality at the cost of an increase in bleeding events. ${ }^{22}$ In MAGELLAN (the Multicenter, Randomized, Parallel Group Efficacy and Safety Study for the Prevention of Venous Thromboembolism in

Table 2 Drugs for prophylaxis of venous thromboembolism in hospitalized medical patients

\begin{tabular}{|c|c|c|}
\hline Drug & Dose & Comment \\
\hline Unfractionated heparin & $\begin{array}{l}5,000 \mathrm{U} \text { subcutaneously, twice or } \\
\text { three times daily* }\end{array}$ & $\begin{array}{l}\text { In critically ill patients, only doses of 5,000 U twice daily } \\
\text { have been studied }\end{array}$ \\
\hline \multicolumn{3}{|l|}{ Low molecular weight heparins } \\
\hline Enoxaparin $\left(\right.$ Lovenox $\left.^{\circledR}\right)$ & $40 \mathrm{mg}$ subcutaneously, once daily & \\
\hline Dalteparin $\left(\right.$ Fragmin $\left.^{\circledR}\right)$ & $5,000 \cup$ subcutaneously, once daily & \\
\hline Tinzaparin $\left(\right.$ Innohep $\left.{ }^{\circledR}\right)$ & $4,500 \cup$ subcutaneously, once daily & \\
\hline Nadroparin (Fraxiparin ${ }^{\circledR}$ ) & $2,850 \cup$ subcutaneously, once daily & \\
\hline Parnaparin (Fluxum ${ }^{\circledast}$ ) & $4,250 \cup$ subcutaneously, once daily & \\
\hline Fondaparinux (Arixtra ${ }^{\circledR}$ ) & $2.5 \mathrm{mg}$ subcutaneously, once daily & $\begin{array}{l}\text { In case of creatinine clearance between } 20 \text { and } 50 \mathrm{~mL} \text { per } \\
\text { minute, lower dose of } 1.5 \mathrm{mg} \text { subcutaneously once daily }\end{array}$ \\
\hline
\end{tabular}

Note: *No head-to-head trials comparing twice daily versus three times daily, but indirect comparisons do not suggest that UFH three times daily dosing, compared with twice daily dosing, reduces VTE or causes more bleeding.

Abbreviations: UFH, unfractionated heparin; VTE, venous thromboembolism. 
Hospitalized Acutely Ill Medical Patients Comparing Rivaroxaban with Enoxaparin), an extended course of rivaroxaban was noninferior at day 10 and superior at days 30-35 with regard to VTE prevention; however, clinically relevant bleeding rates were increased with rivaroxaban up to three-fold. ${ }^{23}$ These findings were confirmed in a pooled analysis involving 14,629 patients, which showed that, compared with a standard course of enoxaparin, prolonged thromboprophylaxis with an oral FXa inhibitor lowered the incidence of thromboembolic events, while doubling the rate of major bleeding both in the long-term and short-term periods. ${ }^{24}$ Due to these safety concerns, use of the new oral anticoagulant drugs, apixaban and rivaroxaban, cannot be currently recommended for VTE prevention in hospitalized medical patients. APEX (the Acute Medically Ill VTE Prevention with Extended Duration Betrixaban Study) is presently evaluating the factor Xa inhibitor, betrixaban, in selected high-risk medically ill patients (ClinicalTrials.gov identifier NCT01583218). The low renal clearance of betrixaban as well as the possibility of dose adjustments are expected to minimize the risk of bleeding with betrixaban. The final data collection date for the primary outcome measure is August 2014.

\section{VTE prophylaxis with fondaparinux}

Fondaparinux is a chemical synthetic pentasaccharide that binds to plasma antithrombin and selectively inhibits factor Xa. Fondaparinux may be administered intravenously or subcutaneously, with time to peak plasma concentrations of less than 2 hours, and a half-life of 17 hours that allows for once-daily subcutaneous administration. Like LMWH, the dose-response effect of fondaparinux is highly predictable, eliminating the need for any dose adjustment or dose monitoring in practice.

Fondaparinux was evaluated for VTE thromboprophylaxis in hospitalized medical patients in a randomized, placebocontrolled, double-blind trial called ARTEMIS (Rixtra for ThromboEmbolism Prevention in a Medical Indications Study). ${ }^{21}$ The ARTEMIS study included 849 acutely ill medical patients 60 years or older who were hospitalized for at least 4 days due to congestive heart failure and/or acute respiratory illness in the presence of chronic lung disease, and/or acute infection or inflammatory disease. Patients were randomized to placebo or $2.5 \mathrm{mg}$ fondaparinux administered subcutaneously once daily for 6 to 14 days. At day 15, VTE occurred in $5.6 \%$ of patients treated with fondaparinux compared with $10.5 \%$ in the placebo group, corresponding to a significant $47 \%$ VTE reduction $(P=0.029)$. Five $(1.5 \%)$ $\mathrm{PE}$, all fatal, occurred in the placebo group, while none was reported with fondaparinux. The incidence of major bleeding was low $(0.2 \%)$ in either study group. Fondaparinux prophylaxis was associated with a lower mortality at day $32(3.3 \%$ versus $6.0 \%$ ), although this difference was not statistically significant.

A recent individual patient data meta-analysis of eight RCTs with over 13,000 hospitalized surgical or medical patients evaluating fondaparinux for the prophylaxis of VTE confirmed a one fifth reduction in mortality with fondaparinux (1.6\%) compared with the control group of placebo or LMWH (2.1\%). ${ }^{25,26}$ Results were consistent irrespective of whether the comparator was placebo $(2.0 \%$ versus $2.6 \%)$ or LMWH (1.5\% versus 1.9\%). Major bleeding occurred more frequently with fondaparinux ( $2.9 \%$ versus $1.88 \%$, odds ratio $1.56 ; 95 \%$ confidence interval 1.24-1.96), and predicted a seven-fold higher risk of death at 30 days $(8.6 \%$ versus $1.7 \%$, adjusted hazard ratio, $6.96 ; 95 \%$ confidence interval 4.60-10.51). However, the pattern of reduced mortality in patients treated with fondaparinux remained consistent irrespective of whether patients experienced major bleeding or not. The survival benefit with fondaparinux, if confirmed, would represent an important finding at variance with the results of analyses on UFH or LMWH that failed to show a significant effect of heparin on survival., ${ }^{3,4,18}$

\section{Duration of VTE prophylaxis}

While thromboprophylaxis is generally continued for 6 to 14 days, or for the duration of hospitalization, ${ }^{2}$ increasing evidence suggests that the risk of VTE may persist after discharge. ${ }^{2,27}$ Subgroups of patients that may remain at higher risk of post-discharge VTE and could benefit from prolonged thromboprophylaxis include for instance those with a marked reduction in mobility, cancer, or prothrombotic conditions such as antiphospholipid syndrome and thrombophilia. Medical patients could therefore benefit from extended thromboprophylaxis, but the potential for (major) bleeding events in a fragile patient, often with numerous comorbidities and comedications, needs to be taken into account. ${ }^{28}$ In a case-control study, extended pharmacologic thromboprophylaxis did not reduce post-discharge symptomatic VTE, and was associated with a higher incidence of major bleeding events. ${ }^{29}$ The randomized, double-blind, placebo-controlled EXCLAIM (Extended Prophylaxis for Venous ThromboEmbolism in Acutely Ill Medical Patients With Prolonged Immobilization) study showed that extended-duration prophylaxis (28 \pm 4 days) with enoxaparin (40 mg once daily), beyond the standard prophylaxis regimen of $10 \pm 4$ days, reduced total VTE events by $38 \%$ compared with placebo $(2.5 \%$ versus $4.0 \%$; absolute risk difference favoring enoxaparin, $-1.53 \%$ 
[95.8\% confidence interval $-2.54 \%$ to $-0.52 \%]$ ) at the cost of a higher rate of major bleeding $(0.8 \%$ versus $0.3 \%$; absolute risk difference favoring placebo, $0.51 \%$ [95\% confidence interval $0.12-0.89]) .{ }^{30}$ The greatest reduction in VTE was observed among elderly patients, patients with cancer, and those with a marked reduction in mobility.

These results, together with those on extended prophylaxis with the new oral anticoagulants, ${ }^{22,23}$ underline the need for a more accurate stratification of medical patients with regard to both the risk of post-discharge VTE and post-discharge bleeding. In a retrospective cohort study, rehospitalization, thrombophilia, cancer, varicose veins, age above 65 years, obesity, acute infection, chronic venous insufficiency, stroke, and heart failure were all associated with post-discharge VTE, whereas male gender, liver disease, blood disease, increasing age, ulcer, rheumatoid arthritis, thrombocytopenia, and thromboembolic stroke were all independent predictors of post-discharge bleeding. ${ }^{31,32}$ The validation of a predictive score for post-discharge VTE and post-discharge bleeding could help identifying subgroups of patients with the greatest benefit-to-risk ratio from extended thromboprophylaxis, and it is therefore eagerly awaited.

\section{Subgroups of patients at higher risk}

Despite evidence and guidelines supporting VTE prevention in hospitalized patients, implementation of VTE prophylaxis remains largely suboptimal, and less than $40 \%$ receive appropriate prophylaxis, with reported rates as low as $18 \%{ }^{33-38}$ The ENDORSE (Epidemiologic International Day for the Evaluation of Patients at Risk for Venous Thromboembolism in the Acute Hospital Care Setting) study was a multinational, cross-sectional survey designed to determine the proportion of at-risk patients from an acute hospital care setting receiving effective prophylaxis. ${ }^{33}$ A total of 68,183 patients were enrolled, with $45 \%$ categorized as surgical and $55 \%$ as medical. Of the $41 \%$ of medical patients judged to be at risk for VTE, only 39\% received appropriate VTE prophylaxis. Several studies have evaluated methods to increase implementation of the guidelines' recommendations into clinical practice, with inconsistent results. Computer-alert programs seemed to increase the use of VTE prophylaxis and reduce the frequency of VTE in hospitalized patients, but this benefit was not confirmed in all studies, and in some the use of the alert system was associated with increased bleeding rates. ${ }^{39-41}$

One of the reasons that may explain the poor implementation of VTE prophylaxis in hospitalized medical patients is the complexity of these patients, who are often elderly and presenting with multiple comorbidities and significant bleeding risk factors, such as renal insufficiency, cancer, and a low platelet count, which may cause reluctance among clinicians to prescribe thromboprophylaxis. These patients are increasingly encountered in clinical practice, but nonetheless were poorly represented in the RCTs. Although the individual RCTs lacked statistical power to detect a significant increase in bleeding among medical patients, pooled analysis confirmed a small but clinically relevant risk associated with the use of prophylactic anticoagulation. ${ }^{18,42}$ The risk of bleeding associated with thromboprophylaxis is expected to be higher in the unselected hospitalized fragile medical patient.

\section{Patients with renal insufficiency}

As many as $40 \%$ of hospitalized medical patients have moderate or severe renal insufficiency. ${ }^{43}$ The use of anticoagulant drugs with a predominant renal clearance, such as fondaparinux, may result in an excessive anticoagulant effect due to drug bioaccumulation, which predisposes to bleeding. In the presence of renal insufficiency, international guidelines suggest to avoid use of drugs that bioaccumulate, and consider using a lower dose or monitoring the drug level or its anticoagulant effect. ${ }^{1}$

A low dose of fondaparinux (1.5 mg once daily) was recently approved for the prevention of VTE in patients with renal insufficiency based on the results of pharmacokinetic simulations in patients undergoing major orthopedic surgery which showed a predicted concentration of the drug with renal impairment similar to that observed with $2.5 \mathrm{mg}$ in patients with normal renal function. The low dose of fondaparinux was evaluated in the FONDAIR study, which included 206 acutely ill medical patients with moderate to severe renal insufficiency, defined as a creatinine clearance of 20-50 mL per minute. ${ }^{44}$ Fondaparinux was given for a mean of 9 days. During the study treatment period, there was one case of major bleeding $(0.49 \%)$ and eight clinically relevant nonmajor bleeds $(3.88 \%)$. Three patients developed symptomatic VTE (1.46\%) and 23 (11\%) died. The FONDAIR study was prematurely interrupted due to the slow recruitment rate. Despite the relatively modest sample size of the study and the lack of a control treatment group, the results of FONDAIR suggest that a low dose of fondaparinux may be a valid and safe option for VTE prophylaxis in a fragile and challenging population, such as the one included in the study where the mean age was 82 years, the mean creatinine clearance was $33 \mathrm{~mL}$ per minute, and over $99 \%$ patients had a Charlson comorbidity score above 5 . 


\section{Patients with cancer}

Acutely ill hospitalized medical patients with cancer may develop asymptomatic or symptomatic VTE in up to $10 \%-30 \%$ of cases, and $\mathrm{PE}$ remains a leading contributor to inhospital death in these patients. ${ }^{45}$ Thus far, no RCT of VTE prophylaxis has included solely hospitalized medical patients with cancer, and data largely derive from subgroup analyses. In the MEDENOX (Prophylaxis in Medical Patients with Enoxaparin Study Group) trial, the incidence of VTE was higher in patients with (18.6\%) compared with those without (10.7\%) previous or current cancer, and thromboprophylaxis with LMWH produced a nonstatistically significant $50 \%$ VTE risk reduction relative to placebo. ${ }^{11}$ Similarly, a post hoc analysis of PREVENT (the Prospective Evaluation of Dalteparin Efficacy for Prevention of VTE in Immobilized Patients Trial) showed a higher incidence of VTE in the subgroup of hospitalized medical cancer patients, and a $63 \%$ reduction in VTE events with LMWH. ${ }^{20}$ Noteworthy is that none of these post hoc analyses reported the risk of bleeding for the subgroup of patients with cancer. Given the increased risk of VTE and an expected low incidence of bleeding complications with prophylactic doses, international guidelines and experts in the field recommend prophylaxis with LMWH, UFH, or fondaparinux in hospitalized medical patients with cancer. ${ }^{45,46}$

\section{Patients with thrombocytopenia}

Thrombocytopenia is a well known risk factor for bleeding events, and in the IMPROVE (International Medical Prevention Registry on Venous Thromboembolism) study, which included over 15,000 acutely ill hospitalized medical patients, a low platelet count at admission was one of the strongest predictors of 14-day inhospital bleeding. ${ }^{28}$ Hospitalized patients with thrombocytopenia were excluded from RCTs of primary VTE prophylaxis, and data on the safety of anticoagulant drugs in these patients are scarce. ${ }^{2}$ The prospective, observational FAITH (Thromboprophylaxis with Fondaparinux of Deep Vein Thrombosis and Pulmonary Embolism in the Acutely-Ill medical Inpatients with Thrombocytopenia) study is presently evaluating the safety and efficacy of fondaparinux in hospitalized medical patients with a platelet count below 100,000/ $\mu \mathrm{L}$ (ClinicalTrials.gov identifier NCT01727401). Fondaparinux (2.5 mg subcutaneously once daily) is given for 6 to 15 days or until discharge, with a lower dose (1.5 mg once daily) for patients with a creatinine clearance of $20-50 \mathrm{~mL}$ per minute. The main outcome of FAITH is the incidence of major bleeding up to 48 hours from the last dose of study drug. The study is currently recruiting patients and final data are expected in 2015.

\section{Patients from intensive care units}

Critically ill hospitalized intensive care unit (ICU) patients are at increased risk of both VTE and bleeding. In the absence of thromboprophylaxis, the reported incidence of VTE in these patients has varied between $15 \%$ and $60 \%,{ }^{47,48}$ although the actual rate may be underestimated since the typical signs and symptoms of VTE are often masked by the patient's clinical condition. The balance of thrombotic to bleeding risk can fluctuate frequently in the ICU, requiring a daily review of thromboprophylaxis prescriptions. Severe renal insufficiency occurs for instance in 20\%-40\% of ICU patients, and could cause bioaccumulation of anticoagulant drugs with a predominant renal excretion, such as LMWH and fondaparinux. These concerns were, however, not substantiated by the results of a study in critically ill patients with severe renal insufficiency receiving daily prophylactic LMWH (dalteparin) who did not appear to have significant heparin bioaccumulation nor excess bleeding. ${ }^{49}$ Vasopressors are commonly used in the ICU and could impair the bioavailability of subcutaneously administered anticoagulant drugs such as LMWH or fondaparinux, potentially reducing the efficacy of prophylaxis. In a study of hemodynamically stable ICU patients who received $2.5 \mathrm{mg}$ fondaparinux subcutaneously, subtherapeutic concentrations of the drug were observed during the first 48 hours. ${ }^{50}$ No data were provided on the incidence of VTE in these patients, leaving it unclear whether these pharmacokinetic changes may influence the risk of VTE. Heparin-based prophylaxis in ICU patients was evaluated versus placebo in three RCTs that failed to demonstrate or exclude a beneficial or detrimental effect of heparin (UFH or LMWH) on symptomatic DVT, symptomatic PE, major bleeding, and mortality. ${ }^{2,47}$ In PROTECT (Prophylaxis for Thromboembolism in Critical Care Trial), a large study conducted in 3,764 ICU patients, LMWH was not superior to UFH with regard to proximal DVT, but reduced PE by half, with similar rates of major bleeding. ${ }^{51}$ Fondaparinux has not been evaluated for VTE prophylaxis in critically ill ICU patients.

For critically ill patients, guidelines suggest the use of LMWH or UFH prophylaxis, substituting mechanical thromboprophylaxis with graduated compression stockings and/or intermittent pneumatic compression for pharmacologic prophylaxis in patients who are bleeding or are at high risk for major bleeding, until the bleeding risk decreases. ${ }^{2}$ 


\section{Patients with heparin-induced thrombocytopenia}

A threatening complication of heparin-based prophylaxis is heparin-induced thrombocytopenia, an adverse immune-mediated drug reaction associated with a high risk of venous and arterial thrombosis, which characteristically develops 5-10 days after initiation of heparin. Heparin exposure leads to the formation of antibodies that recognize and bind to complexes of platelet factor 4 and heparin on the surface of platelets, ultimately resulting in platelet activation and marked thrombin generation. The risk of heparin-induced thrombocytopenia appears to be lower in medical patients $(0.1 \%-1 \%)$ compared with surgical patients ( $1 \%-5 \%)$. UFH and LMWH should not be used in patients with current or previous heparin-induced thrombocytopenia. In hospitalized medical patients with a history of heparin-induced thrombocytopenia who are at high risk of VTE, fondaparinux could be suggested as thromboprophylaxis, although the evidence is essentially limited to case series. ${ }^{52-54}$

\section{Summary}

In summary, VTE prophylaxis with LMWH, UFH, or fondaparinux is recommended for at-risk, acutely ill, hospitalized medical patients who do not present contraindications to pharmacologic prophylaxis, such as active bleeding or high risk for major bleeding. In these latter cases, mechanical prophylaxis with graduated compression stockings or intermittent pneumatic compression may be suggested, although the limited evidence available has failed to demonstrate or exclude a beneficial effect in medical patients. ${ }^{2}$ Thromboprophylaxis should be continued for about 6 to 14 days or until discharge. Some patients could benefit from thromboprophylaxis extended beyond discharge, but major bleeding remains an issue of concern. Predictive scores should be developed to stratify the risk of post-discharge VTE and bleeding to help tailor the duration of prophylaxis. In case of moderate to severe renal insufficiency, low-dose fondaparinux or prophylactic LMWH (dalteparin) may be suggested for VTE prophylaxis, although a formal evaluation in RCTs of the safety of this approach is lacking. In groups of patients at risk for bleeding complications, such as those with cancer or thrombocytopenia, there is the need for additional studies to establish whether the benefits of VTE prophylaxis outweigh the risks.

\section{Disclosure}

The authors report no conflicts of interest in this work.

\section{References}

1. Geerts WH, Bergqvist D, Pineo GF, et al. Prevention of Venous Thromboembolism: American College of Chest Physicians Evidence Based Clinical Practice Guidelines (8th edition). Chest. 2008;133: 381-453.

2. Kahn SR, Lim W, Dunn AS, et al. Prevention of VTE in nonsurgical patients: antithrombotic therapy and prevention of thrombosis: American College of Chest Physicians Evidence-Based Clinical Practice Guidelines (9th edition). Chest. 2012;141(Suppl 2):195S-226S.

3. Dentali F, Douketis JD, Gianni M, Lim W, Crowther MA. Meta-analysis: anticoagulant prophylaxis to prevent symptomatic venous thromboembolism in hospitalized medical patients. Ann Intern Med. 2007;146:278-288.

4. Wein L, Wein S, Haas SJ, Shaw J, Krum H. Pharmacological venous thromboembolism prophylaxis in hospitalized medical patients: a metaanalysis of randomized controlled trials. Arch Intern Med. 2007;167: 1476-1486.

5. Amin AN, Lin J, Thompson S, Wiederkehr D. Real-world rates of in-hospital and post discharge deep-vein thrombosis and pulmonary embolism in at-risk medical patients in the United States. Clin Appl Thromb Hemost. 2011;17:611-619.

6. Francis CW. Prophylaxis for thromboembolism in hospitalized medical patients. N Engl J Med. 2007;356:1438-1444.

7. Simmons AV, Sheppard MA, Cox AF. Deep venous thrombosis after myocardial infarction: predisposing factors. Br Heart J. 1973;35: 623-625.

8. Emerson PA, Marks P. Preventing thromboembolism after myocardial infarction: effect of low-dose heparin or smoking. BMJ. 1977;1: $18-20$.

9. Kelly J, Rudd A, Lewis RR, Coshall C, Moody A, Hunt BJ. Venous thromboembolism after acute ischemic stroke: a prospective study using magnetic resonance direct thrombus imaging. Stroke. 2004;35: 2320-2325.

10. Sherman DG, Albers GW, Bladin C, et al. The efficacy and safety of enoxaparin versus unfractionated heparin for the prevention of venous thromboembolism after acute ischaemic stroke (PREVAIL Study): an open-label randomised comparison. Lancet. 2007;369:1347-1355.

11. Alikhan R, Cohen AT, Combe S, et al. Risk factors for venous thromboembolism in hospitalized patients with acute medical illness: analysis of the MEDENOX Study. Arch Intern Med. 2004;164: 963-968.

12. Grainge MJ, West J, Card TR. Venous thromboembolism during active disease and remission in inflammatory bowel disease: a cohort study. Lancet. 2010;375:657-663.

13. Matta F, Singala R, Yaekoub AY, Najjar R, Stein PD. Risk of venous thromboembolism with rheumatoid arthritis. Thromb Haemost. 2009;101:134-138.

14. Samama MM, Combe S, Conard J, Horellou M-H. Risk assessment models for thromboprophylaxis of medical patients. Thromb Res. 2012;129:127-132.

15. Barbar S, Noventa F, Rossetto V, et al. A risk assessment model for the identification of hospitalized medical patients at risk for venous thromboembolism: the Padua Prediction Score. J Thromb Haemost. 2010;8:2450-2457.

16. Hill J, Treasure T. Reducing the risk of venous thromboembolism in patients admitted to hospital: summary of NICE guidance. BMJ. 2010;340:c95.

17. Dobromirski M, Cohen M. How I manage venous thromboembolism risk in hospitalized medical patients. Blood. 2012;120:1562-1569.

18. Lederle FA, Zylla D, MacDonald R, Wilt TJ. Venous thromboembolism prophylaxis in hospitalized medical patients and those with stroke: a background review for an American College of Physicians clinical practice guideline. Ann Intern Med. 2011;155:602-615.

19. Samama MM, Cohen AT, Darmon JY, et al. A comparison of enoxaparin with placebo for the prevention of venous thromboembolism in acutely ill medical patients. Prophylaxis in Medical Patients with Enoxaparin Study Group. N Engl J Med. 1999;341:793-800. 
20. Leizorovicz A, Cohen AT, Turpie AGG, Olsson CG, Vaitkus PT, Goldhaber SZ. Randomized, placebo-controlled trial of dalteparin for the prevention of venous thromboembolism in acutely ill medical patients. Circulation. 2004;110:874-879.

21. Cohen AT, Davidson BL, Gallus AS, et al. Efficacy and safety of fondaparinux for the prevention of venous thromboembolism in older acute medical patients: randomised placebo controlled trial. $B M J$. 2006;332:325-329.

22. Goldhaber SZ, Leizorovicz A, Kakkar AK, et al. Apixaban versus enoxaparin for thromboprophylaxis in medically ill patients. $N$ Engl $J$ Med. 2011;365:2167-2177.

23. Cohen AT, Spiro TE, Büller HR, et al. Rivaroxaban for thromboprophylaxis in acutely ill medical patients. $N$ Engl J Med. 2013;368: 513-223.

24. Albertsen IE, Larsen TB, Rasmussen LH, Overvad TF, Lip GYH. Prevention of venous thromboembolism with new oral anticoagulants versus standard pharmacological treatment in acute medically ill patients: a systematic review and meta-analysis. Drugs. 2012;72:1755-1764.

25. Eikelboom JW, Quinlan DJ, O’Donnell M. Major bleeding, mortality, and efficacy of fondaparinux in venous thromboembolism prevention trials. Circulation. 2009;120:2006-2011.

26. Eikelboom JW. Effect of fondaparinux $2.5 \mathrm{mg}$ once daily on mortality: a meta-analysis of phase III randomized trials of venous thromboembolism prevention. Eur Heart J Suppl. 2008;10:C8-C13.

27. Spencer FA, Lessard D, Emery C, Reed G, Goldberg RJ. Venous thromboembolism in the outpatient setting. Arch Intern Med. 2007;167: 1471-1475.

28. Decousous H, Tapson VF, Bergmann JF, et al. Factors at admission associated with bleeding risk in medical patients: findings from the IMPROVE investigators. Chest. 2011;139:69-79.

29. Fanikos J, Rao A, Seger AC, et al. Venous thromboembolism prophylaxis for medical service-mostly cancer-patients at hospital discharge. Am J Med. 2011;124:1143-1150.

30. Hull RD, Schellong SM, Tapson VF, et al. Extended-duration venous thromboembolism prophylaxis in acutely ill medical patients with recently reduced mobility: a randomized trial. Ann Intern Med. 2010;153:8-18.

31. Spyropoulos AC, Fisher M, Mahan C, et al. Incidence and risk factors for post-discharge VTE among medically ill patients hospitalized in the United States: 2005-2009. J Thromb Haemost. Conference: 23rd Congress of the International Society on Thrombosis and Haemostasis 57th Annual SSC Meeting Kyoto, Japan. 9; 2011. p. 25.

32. Mahan C, Klaskala W, Fisher M, et al. Risk factors for post-discharge bleeding in US medically ill patients at risk of venous thromboembolism (VTE). J Thromb Haemost. Conference: 23rd Congress of the International Society on Thrombosis and Haemostasis 57th Annual SSC Meeting Kyoto Japan. 9; 2011. p. 410.

33. Cohen AT, Tapson VF, Bergmann J-F, et al. Venous thromboembolism risk and prophylaxis in the acute hospital care setting (ENDORSE study): a multinational cross-sectional study. Lancet. 2008;371:387-394.

34. Bhowmik SS, Biswas S, Kharbanda M, Chatterjee TK, Nandy S. Utilization of DVT prophylaxis in non ICU hospitalized patients. Asian Pacific J Trop Dis. 2012;2 Suppl 2:S707-S711.

35. Pendergraft T, Liu X, Edelsberg J, et al. Prophylaxis against venous thromboembolism in hospitalized medically ill patients. Circ Cardiovasc Qual Outcomes. 2013;6:75-82.

36. Baser O, Sengupta N, Dysinger A, Wang L. Thromboembolism prophylaxis in medical inpatients: effect on outcomes and costs. Am J Manag Care. 2012;18:294-302.
37. Khoury H, Welner S, Kubin M, Folkerts K, Haas S. Disease burden and unmet needs for prevention of venous thromboembolism in medically ill patients in Europe show underutilisation of preventive therapies. Thromb Haemost. 2011;106:600-608.

38. Mokhtari M, Salameh P, Kouchek M, Kashani BS, Taher A, Waked M. The AVAIL ME Extension: a multinational Middle Eastern survey of venous thromboembolism risk and prophylaxis. J Thromb Haemost. 2011;9:1340-1349.

39. Bhalla R, Berger MA, Reissman SH, et al. Improving hospital venous thromboembolism prophylaxis with electronic decision support. $J$ Hosp Med. 2013;8:115-120.

40. Piazza G, Rosenbaum EJ, Pendergast W, et al. Physician alerts to prevent symptomatic venous thromboembolism in hospitalized patients. Circulation. 2009;119:2196-2201.

41. Kucher N, Koo S, Quiroz R, et al. Electronic alerts to prevent venous thromboembolism among hospitalized patients. $N$ Engl $J$ Med. 2005;352:969-977.

42. Lloyd NS, Douketis JD, Moinuddin I, Lim W, Crowther MA. Anticoagulant prophylaxis to prevent asymptomatic deep vein thrombosis in hospitalized medical patients: a systematic review and meta-analysis. J Thromb Haemost. 2008;6:405-414.

43. Dentali F, Riva N, Gianni M, et al. Prevalence of renal failure and use of thromboembolic prophylaxis among medical inpatients at increased risk of venous thromboembolic events. Thromb Res. 2008;123:67-71.

44. Ageno W, Riva N, Noris P, et al. Safety and efficacy of low-dose fondaparinux $(1.5 \mathrm{mg})$ for the prevention of venous thromboembolism in acutely ill medical patients with renal impairment: the FONDAIR study. J Thromb Haemost. 2012;10:2291-2297.

45. Francis CW. Prevention of venous thromboembolism in hospitalized patients with cancer. J Clin Oncol. 2009;27:4874-4880.

46. Farge D, Debourdeau P, Beckers M, et al. International clinical practice guidelines for the treatment and prophylaxis of venous thromboembolism in patients with cancer. $J$ Thromb Haemost. 2013;11:56-70.

47. Chan CM, Shorr AF. Venous thromboembolic disease in the intensive care unit. Semin Respir Crit Care Med. 2010;31:39-46.

48. Cook DJ, Crowther MA. Thromboprophylaxis in the intensive care unit: focus on medical-surgical patients. Crit Care Med. 2010; 38 Suppl:S76-S82.

49. Cook D, Douketis J, Meade M, et al. Venous thromboembolism and bleeding in critically ill patients with severe renal insufficiency receiving dalteparin thromboprophylaxis: prevalence, incidence and risk factors. Crit Care. 2008;12:R32.

50. Cumbo-Nacheli G, Samavati L, Guzman JA. Bioavailability of fondaparinux to critically ill patients. J Crit Care. 2011;26:342-346.

51. The PROTECT Investigators for the Canadian Critical Care Trials Group and the Australian and New Zealand Intensive Care Society Clinical Trials Group. Dalteparin versus unfractionated heparin in critically ill patients. $N$ Engl J Med. 2011;364:1305-1314.

52. Warkentin TE, Pai M, Sheppard JI, Schulman S, Spyropoulos AC, Eikelboom JW. Fondaparinux treatment of acute heparin-induced thrombocytopenia confirmed by the serotonin-release assay: a 30-month, 16-patient case series. J Thromb Haemost. 2011;9:2389-2396.

53. Linkins L-A, Dans AL, Moores LK, et al. Treatment and prevention of heparin-induced thrombocytopenia: Antithrombotic Therapy and Prevention of Thrombosis, 9th ed: American College of Chest Physicians Evidence-based Clinical Practice Guidelines. Chest. 2012;141: e495S-e530S.

54. Kelton JG, Arnold DM, Bates SM. Nonheparin anticoagulants for heparin-induced thrombocytopenia. N Engl J Med. 2013;368:737-744. 


\section{Publish your work in this journal}

Drug Design, Development and Therapy is an international, peerreviewed open-access journal that spans the spectrum of drug design and development through to clinical applications. Clinical outcomes, patient safety, and programs for the development and effective, safe, and sustained use of medicines are a feature of the journal, which

has also been accepted for indexing on PubMed Central. The manuscript management system is completely online and includes a very quick and fair peer-review system, which is all easy to use. Visit http://www.dovepress.com/testimonials.php to read real quotes from published authors.

Submit your manuscript here: http://www.dovepress.com/drug-design-development-and-therapy-journal 